\title{
Impact of the availability of active cytotoxic agents on the survival of patients with advanced gastric cancer
}

\author{
BYUNG HA CHO ${ }^{1}$, HYE SOOK HAN ${ }^{1}$, JHHYUN KWON ${ }^{1}$, JOUNG-HO HAN ${ }^{1}$, SOON MAN YOON ${ }^{1}$, \\ DAE HOON KIM ${ }^{2}$, HYO YUNG YUN ${ }^{2}$, KI HYEONG LEE ${ }^{1}$, SEI JIN YOUN ${ }^{1}$ and SEUNG TAIK KIM ${ }^{1}$ \\ Departments of ${ }^{1}$ Internal Medicine and ${ }^{2}$ Surgery, College of Medicine, Chungbuk National University, \\ Cheongju, North Chungcheong 361-763, Republic of Korea
}

Received August 1, 2014; Accepted April 29, 2015

DOI: $10.3892 / 01.2015 .3564$

\begin{abstract}
Several cytotoxic agents, including fluoropyrimidines, platinums, taxanes and irinotecan, are effective in the treatment of advanced gastric cancer (AGC). However, the effect of the availability of cytotoxic agents on survival has not yet been evaluated. Therefore, the present study assessed the impact of the availability of active cytotoxic agents on the survival of patients with AGC. The records of 216 patients with newly diagnosed AGC that were treated with palliative chemotherapy between March 2002 and November 2012 at Chungbuk National University Hospital were reviewed. For the present study, the patients were divided according to the availability of active cytotoxic agents over the course of treatment: Group 1 received fluoropyrimidine and platinum; group 2 received fluoropyrimidine, platinum and taxane or irinotecan; and group 3 received fluoropyrimidine, platinum, taxane and irinotecan. The median overall survival times for groups 1, 2 and 3 were 6.3, 9.9 and 14.3 months, respectively $(\mathrm{P}<0.0001)$. Multivariate analysis revealed that the Eastern Cooperative Oncology Group (ECOG) performance status and the availability of active cytotoxic agents were independent prognostic factors, as the hazard ratios for mortality were 3.25 for patients with an ECOG performance status of 2-3 [95\% confidence interval (CI), 1.99-5.30; $\mathrm{P}<0.0001$ ], 0.58 for patients in group 2 (95\% CI, $0.42-0.80 ; \mathrm{P}=0.0009)$, and 0.40 for patients in group 3 (95\% CI, $0.28-0.58 ; \mathrm{P}<0.0001)$. The present study reveals that the availability of active cytotoxic agents is associated with an improved survival time in patients with AGC.
\end{abstract}

\section{Introduction}

Gastric cancer is the fourth most frequent malignancy and the second most common cause of cancer-associated mortality

Correspondence to: $\mathrm{Dr}$ Hye Sook Han, Department of Internal Medicine, College of Medicine, Chungbuk National University, 52 Naesudong-ro, Seowon-gu, Cheongju, North Chungcheong 361-763, Republic of Korea

E-mail: sook3529@hanmail.net

Key words: availability, cytotoxic agent, gastric cancer, survival worldwide (1). This cancer is also the second most frequent malignancy in Korea (2). Although patients with early gastric cancer may be successfully treated by surgical resection, the majority of patients experience a relapse subsequent to the first surgical resection (3) or are initially diagnosed with unresectable, locally advanced or metastatic disease (4). For these patients with advanced or metastatic gastric cancer, the objective of treatment is to relieve symptoms, prevent tumor progression and prolong the survival time. Therefore, palliative chemotherapy may play an extremely important role in the treatment of advanced gastric cancer (AGC).

It has been reported that chemotherapy improves the survival of AGC patients in comparison to best supportive care, and it has also been reported that combination chemotherapy is superior to monotherapy in terms of survival, response rate and symptom control (5). In general, fluoropyrimidine, such as 5-fluorouracil (5-FU) or its oral prodrugs, and platinum, such as cisplatin or oxaliplatin, combination regimens are widely accepted as the standard first-line chemotherapy, with a response rate of $25-54 \%$ and a median overall survival time of 8-13 months (5-6). However, more than one-half of the patients with AGC that receive first-line chemotherapy do not demonstrate a response, and even in responders the duration of response may be as short as a few months (6). In addition, the number of patients that maintain a good general condition following the failure of first-line chemotherapy has increased due to the improvements in supportive care. As a result, the number of patients that are good candidates for subsequent salvage chemotherapy has increased. In previous years, novel drugs, including docetaxel, paclitaxel and irinotecan, have been tested in salvage chemotherapy for pre-treated AGC $(7,8)$. With the availability of these active cytotoxic agents, numerous patients with refractory or relapsed gastric cancer subsequent to first-line chemotherapy have received salvage chemotherapy in routine clinical practice, particularly in Asia (8).

Although salvage chemotherapy following first-line treatment may be considered a confirmed option for the treatment of AGC, the impact of the availability of several active cytotoxic agents has not yet been assessed in AGC. Therefore, the present study assessed the impact of the availability of fluoropyrimidines, platinums, taxanes and irinotecan over the course of treatment on the survival of patients with AGC. 


\section{Materials and methods}

Study population. A retrospective chart review was performed on all patients with newly diagnosed locally advanced or metastatic gastric cancer that were treated with standard palliative chemotherapy between March 2002 and November 2012 at the Department of Internal Medicine, Chungbuk National University Hospital (Cheongju, North Chungcheong, Republic of Korea). All patients were consecutive non-selected cases from the Department of Internal Medicine, Chungbuk National University Hospital and all patients were treated outside of clinical trials. Patients were included in the present study if they possessed a histologically-confirmed diagnosis of adenocarcinoma, newly diagnosed locally advanced or metastatic gastric cancer, and medical records containing details of palliative chemotherapy administered. Patients were excluded if they had not received palliative chemotherapy, had received only fluoropyrimidine monotherapy or molecular targeted agents during the course of treatment, had succumbed to AGC during the first hospitalization, or possessed a history of another malignancy. The present study was reviewed and approved by the Institutional Review Board of Chungbuk National University Hospital.

Data collection. The baseline clinical and pathological characteristics at the time of the diagnosis of locally advanced or metastatic gastric cancer were reviewed, including the age, gender, Eastern Cooperative Oncology Group (ECOG) performance status, location of the primary tumor, histological grading according to the World Health Organization (WHO) system, timing of metastatic disease, location of metastasis, number of metastases, baseline hemoglobin and baseline albumin of the patients. Data from medical records on the palliative chemotherapeutic agents administered was also collected, and the patients were divided according to the availability of active cytotoxic agents over the course of treatment, regardless of dose or schedule, as follows: Group 1 received two cytotoxic agents, fluoropyrimidine (5-FU, capecitabine or S-1) and platinum (cisplatin or oxaliplatin); group 2 received three cytotoxic agents, fluoropyrimidine, platinum and taxane (docetaxel or paclitaxel) or irinotecan; and group 3 received four cytotoxic agents, fluoropyrimidine, platinum, taxane and irinotecan.

Statistical analysis. Overall survival was measured from the date of the first administration of first-line chemotherapy to the date of mortality, from any cause, or last follow-up visit. Survival curves were estimated using the Kaplan-Meier method and the survival curves of patients were compared using the log-rank test. A prognostic model for overall survival was constructed using an assessment of variables by univariate analysis followed by multivariate analysis, which was performed using a stepwise Cox proportional hazard regression model. The following variables were included in the univariate analysis: Age, $<65$ years vs. $\geq 65$ years; gender, male vs. female; pre-treatment ECOG performance status, 0-1 vs. 2-3; histological grading according to the WHO system, good (well- or moderately-differentiated) vs. poor (poorly-differentiated or signet ring cell carcinoma) vs. no data; timing of metastatic disease, synchronous vs. metachronous; presence of peritoneal metastases or malignant ascites, present vs. absent; number of metastases, $\leq 1$ vs. 2 vs. $\geq 3$; hemoglobin level, $<10 \mathrm{~g} / \mathrm{dl}$ vs. $\geq 10 \mathrm{~g} / \mathrm{dl}$; and

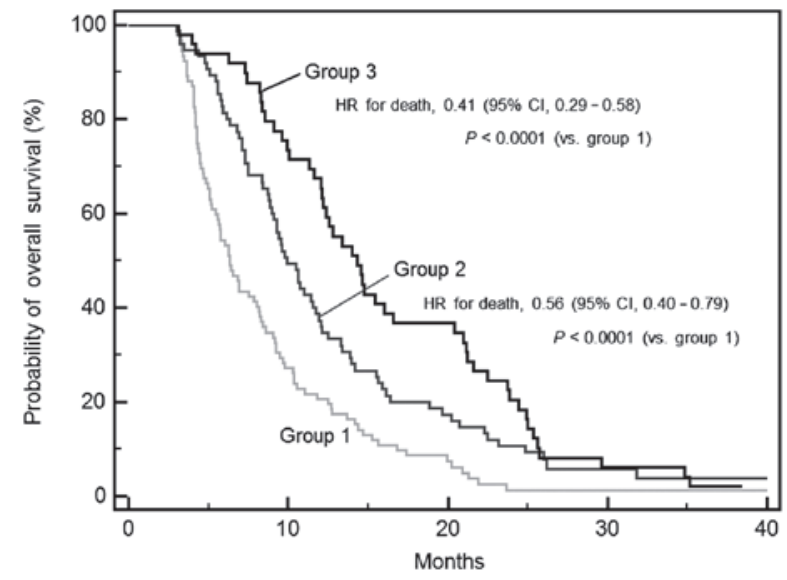

Figure 1. Overall survival according to the availability of active cytotoxic agents. Enrolled patients were divided according to the availability of active cytotoxic agents during the course of treatment, as follows: Group 1 received fluoropyrimidine and platinum; group 2 received fluoropyrimidine, platinum, taxane or irinotecan; and group 3 received fluoropyrimidine, platinum, taxane, and irinotecan. HR, hazard ratio; CI, confidence interval.

albumin level, $<3.5 \mathrm{~g} / \mathrm{dl}$ vs. $\geq 3.5 \mathrm{~g} / \mathrm{dl}$. Hazard ratios (HRs) of the studied outcomes were calculated for each parameter estimate, in addition to the $95 \%$ confidence interval (CI). $\mathrm{P}<0.05$ was considered to indicate a statistically significant difference. All statistical analyses were performed using SPSS software for Windows, version 15.0 (SPSS, Inc., Chicago, IL, USA).

\section{Results}

Baseline patient characteristics. Of the 262 patients with newly-diagnosed locally advanced or metastatic gastric cancer that received palliative chemotherapy between March 2002 and November 2012 at the Department of Internal Medicine, Chungbuk National University Hospital, 216 patients were included in the final analysis. The baseline clinicopathological characteristics of the patients are reported in Table I. The median age was 57 years (range, 18-79 years), and 161 patients (74.5\%) were male. In total, 196 patients (90.7\%) demonstrated good performance status (ECOG status, $0-1$ ). The majority of the patients (97.7\%) possessed metastatic disease, and only five patients (2.3\%) possessed locally advanced disease. The most common metastatic sites were the peritoneum (56.9\%), liver (35.6\%) and distant lymph nodes (31.0\%).

Treatment regimens. The characteristics of the cytotoxic agents used are listed in Table II. The most commonly used cytotoxic agents were 5-FU in 171 patients $(79.2 \%)$, cisplatin in 140 patients (64.8\%) and oxaliplatin in 117 patients (54.2\%). Of the patients enrolled, 92 patients $(42.6 \%)$ were treated with fluoropyrimidine and platinum, classed as group 1, 75 patients (34.7\%) were treated with fluoropyrimidine, platinum and taxane or irinotecan, classed as group 2, and 49 patients (22.7\%) were treated with fluoropyrimidine, platinum, taxane and irinotecan, classed as group 3 , over the course of palliative treatment.

Univariate andmultivariate analysis. The median overall survival time for all patients was 9.3 months (95\% CI, 8.4-10.5 months). The results of the univariate and multivariate analyses for 
Table I. Patient characteristics $(n=216)$.

\begin{tabular}{|c|c|}
\hline Characteristics & Value, n (\%) \\
\hline \multicolumn{2}{|l|}{ Age } \\
\hline Median, years (range) & $57.0(18-79)$ \\
\hline$<65$ years & $126(58.3)$ \\
\hline$\geq 65$ years & $90(41.7)$ \\
\hline \multicolumn{2}{|l|}{ Gender } \\
\hline Male & $161(74.5)$ \\
\hline Female & $55(25.5)$ \\
\hline \multicolumn{2}{|l|}{ ECOG performance status } \\
\hline $0-1$ & $196(90.7)$ \\
\hline $2-3$ & $20(9.3)$ \\
\hline \multicolumn{2}{|c|}{ Location of primary tumor } \\
\hline EGJ and cardia & 25 (11.6) \\
\hline Fundus and body & $87(40.3)$ \\
\hline Antrum & $104(48.1)$ \\
\hline \multicolumn{2}{|l|}{ Histological grade } \\
\hline Good & $72(33.3)$ \\
\hline Poor & $122(56.5)$ \\
\hline No data & $22(10.2)$ \\
\hline \multicolumn{2}{|c|}{$\begin{array}{l}\text { Timing of locally advanced or } \\
\text { metastatic disease }\end{array}$} \\
\hline Synchronous & $139(64.4)$ \\
\hline Metachronous & $77(35.6)$ \\
\hline \multicolumn{2}{|l|}{ Extent of disease } \\
\hline Locally advanced & $5(2.3)$ \\
\hline Metastatic & $211(97.7)$ \\
\hline Liver & 77 (35.6) \\
\hline Peritoneum & $123(56.9)$ \\
\hline Distant lymph nodes & $67(31.0)$ \\
\hline Bone & $22(10.2)$ \\
\hline Lung & $13(6.0)$ \\
\hline \multicolumn{2}{|l|}{ Number of metastases } \\
\hline$\leq 1$ & $98(45.4)$ \\
\hline 2 & $89(41.2)$ \\
\hline$\geq 3$ & $29(13.4)$ \\
\hline \multicolumn{2}{|l|}{ Baseline hemoglobin } \\
\hline Median, g/dl (range) & $11.9(3.5-16.8$ \\
\hline$<10.0 \mathrm{~g} / \mathrm{dl}$ & $59(27.3)$ \\
\hline$\geq 10.0 \mathrm{~g} / \mathrm{dl}$ & $157(72.7)$ \\
\hline \multicolumn{2}{|l|}{ Baseline albumin } \\
\hline Median, g/dl (range) & $3.9(2.4-5.0)$ \\
\hline$<3.5 \mathrm{~g} / \mathrm{dl}$ & $42(19.4)$ \\
\hline$\geq 3.5 \mathrm{~g} / \mathrm{dl}$ & $174(80.6)$ \\
\hline
\end{tabular}

ECOG, Eastern Cooperative Oncology Group performance status; EGJ, esophago-gastric junction.

overall survival are summarized in Table III. Univariate analysis revealed that the ECOG performance status (0-1 vs. 2-3) and the availability of the active cytotoxic agents (group 1 vs. group 2 vs. group 3) had prognostic significance. The median overall
Table II. Characteristics of palliative chemotherapy $(n=216)$.

\begin{tabular}{lr}
\hline Characteristics & Value, n (\%) \\
\hline Cytotoxic agents & \\
Fluoropyrimidines & $171(79.2)$ \\
5-FU & $70(32.4)$ \\
Capecitabine & $51(23.6)$ \\
S-1 & \\
Platinum & $140(64.8)$ \\
Cisplatin & $117(54.2)$ \\
Oxaliplatin & \\
Taxanes & $55(25.5)$ \\
Docetaxel & $45(20.8)$ \\
Paclitaxel & $74(34.3)$ \\
Irinotecan & \\
Availability of active & \\
cytotoxic agents & $92(42.6)$ \\
Group 1 & $75(34.7)$ \\
Group 2 & $49(22.7)$ \\
Group 3 & \\
\hline
\end{tabular}

5-FU, 5-Fluorouracil.

survival time was significantly longer in patients with an ECOG performance status of 0-1 compared with in patients with an ECOG performance status of 2-3 (9.9 vs. 4.7 months, respectively; HR for mortality, 3.52; 95\% CI, 1.57-7.86; $\mathrm{P}<0.0001$ ). The median overall survival times were 6.3 months in group 1 , 9.9 months in group 2, and 14.3 months in group 3; these differences were statistically significant $(\mathrm{P}<0.0001)$. The estimated HRs for mortality were 0.56 for group 2 (95\% CI, 0.40-0.79) and 0.41 for group 3 (95\% CI, 0.25-0.58; Fig. 1).

A multivariate Cox proportional hazard model revealed that the ECOG performance status and the availability of active cytotoxic agents were independent prognostic factors for AGC outcome (Table III). The estimated HRs for mortality in patients with an ECOG performance status of 2-3 compared to patients with an ECOG performance status of $0-1$ was 3.25 (95\% CI, 1.99-5.30; $\mathrm{P}<0.0001)$. The estimated HRs for mortality in groups 2 and 3 compared to group 1 were 0.58 (95\% CI, 0.42-0.80; $\mathrm{P}=0.0009)$ and $0.40(95 \% \mathrm{CI}$, $0.28-0.58 ; \mathrm{P}<0.0001)$, respectively.

\section{Discussion}

The present study retrospectively analyzed data obtained from 216 patients with AGC that had undergone palliative chemotherapy with active cytotoxic agents, such as fluoropyrimidines and platinums or taxanes or irinotecan. The present analysis demonstrated that the availability of these active cytotoxic agents in the course of treatment had a positive impact on the survival of patients with AGC. In the present study, 92 patients $(42.6 \%)$ were treated with fluoropyrimidine and platinum only, while 75 patients $(34.7 \%)$ received one more cytotoxic agent, irinotecan or a taxane, 
Table III. Univariate and multivariate analyses of overall survival.

\begin{tabular}{|c|c|c|c|c|c|c|c|c|}
\hline \multirow[b]{2}{*}{ Characteristics } & \multirow[b]{2}{*}{ Total, $\mathrm{n}$} & \multicolumn{4}{|c|}{ Univariate analysis } & \multicolumn{3}{|c|}{ Multivariate analysis } \\
\hline & & $\begin{array}{c}\text { Median OS, } \\
\text { months }(95 \% \mathrm{CI})\end{array}$ & HR & $95 \% \mathrm{CI}$ & P-value & HR & $95 \% \mathrm{CI}$ & P-value \\
\hline \multicolumn{9}{|l|}{ Age } \\
\hline$<65$ years & 126 & $9.3(8.3-11.0)$ & & & 0.985 & & & \\
\hline$\geq 65$ years & 90 & $9.7(8.2-11.0)$ & 1.00 & $0.76-1.31$ & & & & \\
\hline \multicolumn{9}{|l|}{ Gender } \\
\hline Male & 161 & $9.4(8.4-10.5)$ & & & 0.648 & & & \\
\hline Female & 55 & $9.3(7.5-11.8)$ & 0.93 & $0.68-1.28$ & & & & \\
\hline \multicolumn{9}{|c|}{ ECOG performance status } \\
\hline $0-1$ & 196 & $9.9(9.1-11.4)$ & & & $<0.001$ & & & \\
\hline $2-3$ & 20 & $4.7(3.9-6.9)$ & 3.52 & $1.57-7.86$ & & 3.25 & $1.99-5.30$ & $<0.0001$ \\
\hline \multicolumn{9}{|c|}{ Histological grade } \\
\hline Good & 72 & $10.3(8.7-13.7)$ & & & 0.087 & & & \\
\hline Poor & 122 & $8.4(7.3-9.8)$ & 1.36 & $0.76-1.31$ & & & & \\
\hline No data & 22 & $11.6(9.4-20.2)$ & 1.03 & $0.66-1.60$ & & & & \\
\hline \multicolumn{9}{|c|}{$\begin{array}{l}\text { Timing of locally advanced } \\
\text { or metastatic disease }\end{array}$} \\
\hline Synchronous & 139 & $9.6(8.6-10.6)$ & & & 0.551 & & & \\
\hline Metachronous & 77 & $9.1(6.3-11.8)$ & 1.09 & $0.83-1.44$ & & & & \\
\hline \multicolumn{9}{|c|}{ Peritoneal metastases } \\
\hline Yes & 123 & $9.5(8.2-10.8)$ & & & 0.174 & & & \\
\hline No & 93 & $9.3(8.3-11.8)$ & 1.21 & $0.92-1.58$ & & & & \\
\hline \multicolumn{9}{|c|}{ Number of metastases } \\
\hline$\leq 1$ & 98 & $9.3(7.5-10.8)$ & & & 0.261 & & & \\
\hline 2 & 89 & $9.3(8.3-11.0)$ & 1.23 & $0.92-1.66$ & & & & \\
\hline$\geq 3$ & 29 & $9.9(7.1-14.8)$ & 0.95 & $0.64-1.41$ & & & & \\
\hline \multicolumn{9}{|c|}{ Baseline hemoglobin } \\
\hline$<10.0 \mathrm{~g} / \mathrm{dl}$ & 59 & $8.9(6.9-10.0)$ & & & 0.641 & & & \\
\hline$\geq 10.0 \mathrm{~g} / \mathrm{dl}$ & 157 & $9.7(8.6-11.3)$ & 0.93 & $0.68-1.27$ & & & & \\
\hline \multicolumn{9}{|c|}{ Baseline albumin } \\
\hline$<3.5 \mathrm{~g} / \mathrm{dl}$ & 42 & $9.1(6.9-11.6)$ & & & 0.875 & & & \\
\hline$\geq 3.5 \mathrm{~g} / \mathrm{dl}$ & 174 & $9.4(8.4-10.8)$ & 0.97 & $0.69-1.37$ & & & & \\
\hline \multicolumn{9}{|c|}{$\begin{array}{l}\text { Availability of active } \\
\text { cytotoxic agents }\end{array}$} \\
\hline Group 1 & 92 & $6.3(5.5-8.2)$ & & & $<0.001$ & & & \\
\hline Group 2 & 75 & $9.9(9.0-11.8)$ & 0.56 & $0.40-0.79$ & & 0.58 & $0.42-0.80$ & 0.0009 \\
\hline Group 3 & 49 & $14.3(12.1-20.4)$ & 0.41 & $0.29-0.58$ & & 0.40 & $0.28-0.58$ & $<0.0001$ \\
\hline
\end{tabular}

OS, overall survival; HR, hazard ratio; CI, confidence interval; ECOG, Eastern Cooperative Oncology Group performance status; 5-FU, 5-Fluorouracil.

and 49 patients $(22.7 \%)$ received all four active cytotoxic agents. Multivariate analysis revealed that the availability of more active cytotoxic drugs was an independent prognostic factor for survival compared with fluoropyrimidine and platinum only. This finding suggests that it is important to use all cytotoxic agents that have well-demonstrated clinical activity in AGC to guarantee the maximal benefit of systemic therapy for overall survival in patients with AGC.
Although the median survival time of patients with AGC remained below one year, numerous cytotoxic agents have been investigated over the previous decades, including fluoropyrimidines, which are administered orally or intravenously, anthracyclines, cisplatin, oxaliplatin, taxanes and irinotecan (5-7). Currently, various targeted agents are being tested in clinical trials and promising data have been recently published for trastuzumab-containing therapy, with median survival time exceeding 
one year (9). Novel oral fluoropyrimidines, including capecitabine and $\mathrm{S}-1$, are not clinically inferior to 5-FU in terms of survival, and additional advantages of novel oral fluoropyrimidines include the convenience of oral chemotherapy, which avoids the potential morbidity associated with central venous access, and the opportunity to make simple dose adjustments to the oral agent during the treatment cycle to manage toxicity (10-13). Cisplatin has been an integral component of AGC reference regimens (6). However, oxaliplatin has been extensively studied in AGC due to the specific side-effects of cisplatin, including nephrotoxicity, emetogenicity and ototoxicity. Due to the non-inferior efficacy, oxaliplatin may be substituted for cisplatin in the treatment of AGC, and elderly patients may derive a particular benefit from treatment with oxaliplatin instead of cisplatin $(10,14)$. Taxanes, such as docetaxel or paclitaxel, which bind and stabilize microtubules and therefore lead to cell-cycle arrest, have also been used as a first-line therapy for AGC $(15,16)$. In addition, irinotecan has been reported to demonstrate activity in gastrointestinal cancers, and irinotecan-based combination regimens have been studied as a first-line alternative to platinum-based chemotherapy $(17,18)$. The availability of these active cytotoxic agents opened the option of sequential salvage chemotherapy in AGC patients.

Second-line chemotherapy is currently considered to be a standard therapy option for patients that demonstrate disease progression during or subsequent to first-line chemotherapy. Docetaxel and irinotecan have been evaluated extensively for second-line therapy in patients for whom fluoropyrimidine and platinum have failed (19). Three randomized controlled trials have revealed the increased survival of patients administered with either docetaxel or irinotecan monotherapy compared with those receiving best supportive care (20-22). As the majority of patients with AGC are initially treated with fluoropyrimidine and platinum, it appears more prudent to avoid these drugs in second-line regimens for these patients. No statistically significant differences were observed between the overall survival, progression-free survival and response rates for patients receiving taxanes and those receiving irinotecan $(19,23)$. Thus, either taxanes or irinotecan may be recommended as a treatment option for second-line chemotherapy in patients with AGC. Although evidence is limited with regard to the efficacy of third-line chemotherapy in AGC, this therapy may have contributed to the prolonged overall survival time. Several studies have demonstrated that third-line chemotherapy performs better compared with best supportive care in patients with AGC in terms of overall survival and quality of life (24-26). The sequence of second and third-line regimens, including taxanes and irinotecan, did not present any significant difference in overall survival or time to progression subsequent to the failure of fluoropyrimidine and platinum chemotherapy (24). The differences in toxicity profiles, previous chemotherapy agents, and treatment schedules between the two treatments may aid in choosing between taxanes or irinotecan.

The current results suggest that the use of all active cytotoxic agents improves the overall survival in patients with AGC. However, if sequential treatment with all active cytotoxic agents cannot be guaranteed for $100 \%$ of the patients, the use of a triplet combination protocol may be considered as first-line therapy. The safety and efficacy of this approach has been assessed in previous clinical trials $(15,16)$. Triplet combination chemotherapy comprising an anthracycline or a taxane in addition to fluoropyrimidine and platinum compounds has resulted in higher response rates and a modest improvement in overall survival compared with doublet combinations (6). In the majority of European countries, the epirubicin, cisplatin and fluorouracil (ECF) regimen is more commonly used, based on a phase 3 randomized trial that compared the administration of the ECF regimen with the administration of fluorouracil, doxorubicin and methotrexate (27). The docetaxel, cisplatin and fluorouracil (DCF) regimen has previously been tested in the V325 phase 3 trial. In this trial, it was found that the DCF regimen not only significantly improved the clinical benefit of chemotherapy, but also improved the quality of life, time to progression and overall survival compared with cisplatin and fluorouracil without docetaxel (15). However, the high rate of treatment-associated toxicity limits the applicability of this regimen to all patients, particularly those that are elderly or have a poor performance status. Therefore, several modifications to the schedule of triplet combination chemotherapy or growth factor support have been investigated in an attempt to minimize the toxicity that occurs with this regimen $(28,29)$.

Although the benefit of sequential salvage chemotherapy is evident, the disease control rate is $30-40 \%$. This indicates that more than one-half of patients do not benefit from salvage chemotherapy and suffer from toxicities. Therefore, it is important to predict whether patients may benefit from sequential salvage chemotherapy. Previous studies have indicated that several factors should be considered in order to assess the response to sequential salvage chemotherapy, such as the performance status of the patient, extent of disease (locally advanced or metastatic), cumulative toxicity, lack of cross-resistance of the tumor cells to previously used drugs and progression-free survival of the patient following previous chemotherapy $(26,30)$. Therefore, predictive factors for the potential survival benefit of salvage chemotherapy with active cytotoxic agents require additional investigation to avoid the development of toxic effects in patients that are unlikely to benefit from the therapy.

The present study demonstrates several limitations. Firstly, it is a retrospective analysis. However, all patients were consecutive non-selected cases that received chemotherapy treatment outside clinical trials and were followed by the Department of Internal Medicine, Chungbuk National University Hospital under the supervision of the same oncology team, which also addresses real-life situations. Secondly, a shorter overall survival time was demonstrated in the present study compared with previous studies. This is due to the population in the present study possessing a poor prognosis, with $97.7 \%$ of the patients experiencing metastatic cancer, $56.1 \%$ possessing peritoneal carcinomatosis and $9.3 \%$ demonstrating an ECOG performance status of 2-3.

In conclusion, the present study suggests that the availability of active cytotoxic agents in the course of treatment is associated with improved survival in patients with AGC. Additional prospective studies of effective administration schedules for patients receiving all active cytotoxic agents, in addition to studies investigating the factors that predict the survival benefit from salvage chemotherapy should continue in patients with AGC. 


\section{Acknowledgements}

This study was supported by a Basic Science Research Program through the National Research Foundation of Korea, funded by the Ministry of Education, Science, and Technology (grant no., 2007-0054930).

\section{References}

1. Jemal A, Bray F, Center MM, Ferlay J, Ward E and Forman D: Global cancer statistics. CA Cancer J Clin 61: 69-90, 2011.

2. Jung KW, Won YJ, Kong HJ, Oh CM, Seo HG and Lee JS: Cancer statistics in Korea: Incidence, mortality, survival and prevalence in 2010. Cancer Res Treat 45: 1-14, 2013.

3. D'Angelica M, Gonen M, Brennan MF, et al: Patterns of initial recurrence in completely resected gastric adenocarcinoma. Ann Surg 240: 808-816, 2004.

4. Macdonald JS: Gastric cancer - new therapeutic options. N Engl J Med 355: 76-77, 2006

5. Wagner AD, Grothe W, Haerting J, et al: Chemotherapy in advanced gastric cancer: A systematic review and meta-analysis based on aggregate data. J Clin Oncol 24: 2903-2909, 2006.

6. Wagner AD, Unverzagt S, Grothe W, et al: Chemotherapy for advanced gastric cancer. Cochrane Database Syst Rev: CD004064, 2010

7. Wesolowski R, Lee C and Kim R: Is there a role for second-line chemotherapy in advanced gastric cancer? Lancet Oncol 10: 903-912, 2009.

8. Baek SK, Kim SY, Jeong JH, Cho KS and Yoon HJ: Second-line chemotherapy for advanced gastric cancer in Korea. Gastric Cancer 15: 345-354, 2012.

9. Bang YJ, Van Cutsem E, Feyereislova A, et al; ToGA Trial Investigators: Trastuzumab in combination with chemotherapy versus chemotherapy alone for treatment of HER2-positive advanced gastric or gastro-oesophageal junction cancer (ToGA): A phase 3, open-label, randomised controlled trial. Lancet 376: 687-697, 2010.

10. Cunningham D, Starling N, Rao S, Iveson T, Nicolson M, Coxon F, Middleton G, Daniel F, Oates J and Norman AR; Upper Gastrointestinal Clinical Studies Group of the National Cancer Research Institute of the United Kingdom. Capecitabine and oxaliplatin for advanced esophagogastric cancer. N Engl J Med 358: 36-46, 2008.

11. Kang YK, Kang WK, Shin DB, Chen J, Xiong J, Wang J, Lichinitser M, Guan Z, Khasanov R, Zheng L, Philco-Salas M, et al: Capecitabine/cisplatin versus 5 -fluorouracil/cisplatin as first-line therapy in patients with advanced gastric cancer: A randomised phase III noninferiority trial. Ann Oncol 20: 666-673, 2009.

12. Koizumi W, Narahara H,Hara T, Takagane A, Akiya T, Takagi M, Miyashita K, Nishizaki T, Kobayashi O, Takiyama W, Toh Y, et al: S-1 plus cisplatin versus S-1 alone for first-line treatment of advanced gastric cancer (SPIRITS trial): A phase III trial. Lancet Oncol 9: 215-221, 2008

13. Boku N, Yamamoto S, Fukuda H, Shirao K, Doi T, Sawaki A, Koizumi W, Saito H, Yamaguchi K, Takiuchi H, Nasu J and Ohtsu A; Gastrointestinal Oncology Study Group of the Japan Clinical Oncology Group. Fluorouracil versus combination of irinotecan plus cisplatin versus S-1 in metastatic gastric cancer: A randomised phase 3 study. Lancet Oncol 10: 1063-1069, 2009.

14. Al-Batran SE, Hartmann JT, Probst S, Schmalenberg H, Hollerbach S, Hofheinz R, Rethwisch V, Seipelt G, Homann N, Wilhelm G, Schuch G, et al; Arbeitsgemeinschaft Internistische Onkologie. Phase III trial in metastatic gastroesophageal adenocarcinoma with fluorouracil, leucovorin plus either oxaliplatin or cisplatin: A study of the Arbeitsgemeinschaft Internistische Onkologie. J Clin Oncol 26: 1435-1442, 2008.

15. Van Cutsem E, Moiseyenko VM, Tjulandin S, Majlis A, Constenla M, Boni C, Rodrigues A, Fodor M, Chao Y, Voznyi E, Risse ML and Ajani JA; V325 study group. Phase III study of docetaxel and cisplatin plus fluorouracil compared with cisplatin and fluorouracil as first-line therapy for advanced gastric cancer: A report of the V325 study group. J Clin Oncol 24: 4991-4997, 2006.
16. Chen XL, Chen XZ, Yang C, Liao YB, Li H, Wang L, Yang K, Li K, Hu JK, Zhang B, Chen ZX, et al: Docetaxel, cisplatin and fluorouracil (DCF) regimen compared with non-taxane-containing palliative chemotherapy for gastric carcinoma: A systematic review and meta-analysis. PLoS One 8: e60320, 2013.

17. Dank M, Zaluski J, Barone C, Valvere V, Yalcin S, Peschel C, Wenczl M, Goker E, Cisar L, Wang K and Bugat R: Randomized phase III study comparing irinotecan combined with 5-fluorouracil and folinic acid to cisplatin combined with 5-fluorouracil in chemotherapy naive patients with advanced adenocarcinoma of the stomach or esophagogastric junction. Ann Oncol 19: 1450-1457, 2008.

18. Moehler M, Kanzler S, Geissler M, Raedle J, Ebert MP, Daum S, Flieger D, Seufferlein T, Galle PR and Hoehler T; Arbeitsgemeinschaft Internistische Onkologie, Germany. A randomized multicenter phase II study comparing capecitabine with irinotecan or cisplatin in metastatic adenocarcinoma of the stomach or esophagogastric junction. Ann Oncol 21: 71-77, 2010.

19. Kim HS, Kim HJ, Kim SY, Kim TY, Lee KW, Baek SK, Kim TY, Ryu MH, Nam BH and Zang DY: Second-line chemotherapy versus supportive cancer treatment in advanced gastric cancer: A meta-analysis. Ann Oncol 24: 2850-2854, 2013.

20. Thuss-Patience PC, Kretzschmar A, Bichev D, Deist T, Hinke A, Breithaupt K, Dogan Y, Gebauer B, Schumacher G and Reichardt P: Survival advantage for irinotecan versus best supportive care as second-line chemotherapy in gastric cancer - a randomised phase III study of the Arbeitsgemeinschaft Internistische Onkologie (AIO). Eur J Cancer 47: 2306-2314, 2011.

21. Kang JH,Lee SI, Lim do H, Park KW, Oh SY, Kwon HC, Hwang IG, Lee SC, Nam E, Shin DB, Lee J, et al: Salvage chemotherapy for pretreated gastric cancer: A randomized phase III trial comparing chemotherapy plus best supportive care with best supportive care alone. J Clin Oncol 30: 1513-1518, 2012.

22. Ford HE, Marshall A, Bridgewater JA, Janowitz T, Coxon FY, Wadsley J, Mansoor W, Fyfe D, Madhusudan S, Middleton GW, Swinson D, et al; COUGAR-02 Investigators: Docetaxel versus active symptom control for refractory oesophagogastric adenocarcinoma (COUGAR-02): An open-label, phase 3 randomised controlled trial. Lancet Oncol 15: 78-86, 2014.

23. Hironaka S, Ueda S, Yasui H, et al: Randomized, open-label, phase III study comparing irinotecan with paclitaxel in patients with advanced gastric cancer without severe peritoneal metastasis after failure of prior combination chemotherapy using fluoropyrimidine plus platinum: WJOG 4007 trial. J Clin Oncol 31: 4438-4444, 2013.

24. Park JS, Lim JY, Park SK, Kim MK, Ko HS, Yoon SO, Kim JW, Choi SH and Cho JY: Prognostic factors of second and third line chemotherapy using 5-fu with platinum, irinotecan and taxane for advanced gastric cancer. Cancer Res Treat 43: 236-243, 2011.

25. Shim HJ, Yun JY, Hwang JE, Bae WK, Cho SH and Chung IJ: Prognostic factor analysis of third-line chemotherapy in patients with advanced gastric cancer. Gastric Cancer 14: 249-256, 2011.

26. Lee MJ, Hwang IG, Jang JS, Choi JH, Park BB, Chang MH, Kim ST, Park SH, Kang MH and Kang JH: Outcomes of third-line docetaxel-based chemotherapy in advanced gastric cancer who failed previous oxaliplatin-based and irinotecan-based chemotherapies. Cancer Res Treat 44: 235-241, 2012.

27. Webb A, Cunningham D, Scarffe JH, Harper P, Norman A, Joffe JK, Hughes M, Mansi J, Findlay M, Hill A, Oates J, et al: Randomized trial comparing epirubicin, cisplatin, and fluorouracil versus fluorouracil, doxorubicin and methotrexate in advanced esophagogastric cancer. J Clin Oncol 15: 261-267, 1997.

28. Overman MJ, Kazmi SM, Jhamb J, Lin E, Yao JC, Abbruzzese JL, Ho L, Ajani J and Phan A: Weekly docetaxel, cisplatin and 5-fluorouracil as initial therapy for patients with advanced gastric and esophageal cancer. Cancer 116: 1446-1453, 2010.

29. Polyzos A, Felekouras E, Karatzas T, Griniatsos J, Dimitroulis D, Polyzos K, Kontzoglou K, Mantas D, Karavokyros J, Nikiteas N, Tsavaris N, et al: Modified docetaxel-cisplatin in combination with capecitabine as first-line treatment in metastatic gastric cancer. A phase II study. Anticancer Res 32: 4151-4156, 2012.

30. Kanagavel D, Pokataev IA, Fedyanin MY, Tryakin AA, Bazin IS, Narimanov MN, Yakovleva ES, Garin AM and Tjulandin SA: A prognostic model in patients treated for metastatic gastric cancer with second-line chemotherapy. Ann Oncol 21: 1779-1785, 2010. 